and

are identical. Hence

$$
\frac{\partial \omega}{\partial y} y^{\prime}+\frac{\partial \omega}{\partial x}=0
$$

$$
\xi \frac{\partial \omega}{\partial x}+\eta \frac{\partial \omega}{\partial y}=\chi\left(\omega_{1}\right)=F(\omega) .
$$

University of Missouri, November, 1909.

\title{
ON THE DISCONTINUOUS $\zeta$-GROUPS DEFINED BY RATIONAL NORMAL CURVES IN A SPACE OF $n$ DIMENSIONS.
}

BY PROFIGSSOR J. W. YOUNG.

(Read before the Chicago Section of the American Mathematical Society, January 1, 1910.)

THE present note completes in an important particular a paper* which I presented to the Society some two years ago. I there considered the discontinuous groups $\Gamma_{n}$ of linear fractional transformations

$$
\zeta^{\prime}=\begin{aligned}
& \alpha \zeta+\beta \\
& \gamma \zeta+\delta
\end{aligned}
$$

on the complex variable $\zeta$, defined as follows by a rational normal curve $C_{n}$ in a space $S_{n}$ of $n$ dimensions : The given $C_{n}$ is transformed into itself by a group of $\infty^{3}$ collineations

$$
z_{i}^{\prime}=\sum_{k=0}^{n} a_{i k_{k} z_{k}} \quad(i=0,1, \ldots, n)
$$

in $S_{n}$. Each of these collineations subjects the parameter $\zeta$ of the points of $C_{n}$ to a substitution (1), so that the continuous three-parameter groups of transformations (1) and (2) are simply isomorphic. If now the transformations (2) be restricted to those whose coefficients $a_{i k}$ are rational integers with determinant $\left|a_{i k_{f}}\right|=1$, the resulting subgroup of the three-parameter group of transformations (2) will be properly discontinuous.

* " A fundamental invariant of the discontinuous $\zeta$-groups defined by the normal curves of order $n$ in a space of $n$ dimensions," BuLceTrN, vol. 14 (1908), pp. 363-367. 
The same is then true of the group of transformations (1) which corresponds in the isomorphism already noted to the discontinuous subgroup just defined.

In the paper cited above I proved the following theorem :

In the discontinuous $\zeta$-groups defined as indicated by any normal curve $C_{n}$ in a space $S_{n}$ the period $\omega$ of any elliptic substitution must satisfy a relation of the form

$$
\sin \frac{(n+1) \pi}{\omega}=J \sin \frac{\pi}{\omega},
$$

where $J$ is an integer (positive, negative, or zero).

This theorem gives a necessary condition on the positive integers $\omega$ that may be periods of elliptic substitutions in any $\Gamma_{n}$. In particular, the numbers $n, n+1, n+2$ are all possible values of $\omega$ under this condition. It has not been shown hitherto, however, that groups $\Gamma_{n}$ really exist containing substitutions of these periods. In the present note it is shown how to construct a $C_{n}$ which will define in the manner indicated a group $\Gamma_{n}$ containing any given substitution of period $n+1$ or $n+2$. The solution $\omega=n$ is not in general possible when $n>2$.

We recall first the necessary and sufficient condition that a collineation in $S_{n}$ leave invariant a normal $C_{n}{ }^{*}$ We will suppose that the collineation produces on the $C_{n}$ a projectivity with two distinct double points, $P_{0}$ and $P_{n}$. The osculating $k$-spaces $(k=1, \ldots, n-1)$ at $P_{0}$ and $P_{n}{ }^{n}$ are then invariant; and the osculating $k$-space at $P_{0}$ determines with the osculating $(n-k)$-space at $P_{n}$ a double point $P_{k}$ of the collineation.

The invariant configuration of the collineation is then a complete $(n+1)$-point in $S_{n}, \dagger$ two of whose vertices are on the curve and which is completely determined by these two points and the curve. An $(n+1)$-point thus situated with reference to a $C_{n}$ will be said to osculate the $C_{n}$ at the two points. If this invariant $(n+1)$-point is taken as the fundamental $(n+1)$ point of the coordinate system, the points $(0,0, \ldots, 1)$ and $(1,0, \ldots, 0)$ being taken as $P_{0}$ and $P_{n}$ respectively, the equations of the $C_{n}$ are of the form

* Gino Loria, "Sulle curve razionali normali in un spazio an $n$ dimensioni," Giornale di Matematiche, vol. 26 (1888), p. 345.

+ It may, of course, happen that more than $n+1$ points are left invariant by the collineation. But in any case the invariant configuration must include a complete $(n+1)$-point of the specified kind. 


$$
z_{i}=c_{i} \zeta_{i}^{n-\imath} \quad(i=0,1, \cdots, n),
$$

where the $c_{i}$ are any constants.

It now follows readily that the roots of the characteristic equation of any collineation leaving the $C_{n}$ invariant must be proportional to the powers $\mu^{n}, \mu^{n-1}, \cdots, \mu, 1$ of a number $\mu$. But this condition with the preceding is at once seen to be also sufficient. We have then

Theorem 1. The necessary and sufficient condition that $\alpha$ collineation in $S_{n}$ (with at least two distinct double points *) leave a rational normal curve $C_{n}$ invariant is that its invariant configuration contain a complete $(n+1)$-point and that the roots $\lambda$ of its characteristic equation be proportional to the powers $\mu^{n}, \mu^{n-1}$, $\ldots, \mu, 1$ of a number $\mu . \quad A$ collineation satisfying this condition leaves every normal curve invariant which is osculated by the invariant $(n+1)$-point at the points $\lambda=\rho \mu^{n}$ and $\lambda=\rho$.

We are now in a position to write down the equations of any $C_{n}$ which is left invariant by a given collineation, if such $C_{n}^{\text {'s }}$ exist. Let $\pi$ be any collineation satisfying the condition of Theorem 1. Let

$$
Z_{0}=0, \quad Z_{1}=0, \quad \cdots, \quad Z_{n}=0
$$

be the equations the $n+1$ invariant $(n-1)$-spaces of the invariant $(n+1)$-point of $\pi$. The collineation may then be written

$$
\rho Z_{i}^{\prime}=\mu^{n-i} Z_{i} \quad(i=0,1, \cdots, n) .
$$

Any $C_{n}$ left invariant by $\pi$ is then given by the equations

$$
Z_{i}=c_{i} \zeta^{n-i} \quad(i=0,1, \ldots, n) .
$$

To complete the proof of the existence theorem which we have in view it is necessary only to show that collineations of periods $n+2$ and $n+1$ with integral coefficients and determinant 1 exist in $S_{n}$ which satisfy the conditions of Theorem 1 . Representing a collineation by the matrix of its coefficients we find readily that the two collineations

* It is readily seen that, if the collineation has two distinct double points, it must have at least $n+1$, two of which are on the curve. Collineations with only a single double point and leaving a $C_{n}$ invariant exist, moreover ; a fact which was overlooked by Loria in the paper quoted above. Cf. in this connection the latter part of the present paper. 


$$
\pi_{1}=\left|\begin{array}{rrrrrrr}
0 & 1 & 0 & 0 & \cdots & 0 & 0 \\
0 & 0 & 1 & 0 & \cdots & 0 & 0 \\
\vdots & \vdots & \vdots & \vdots & \cdots & \vdots & \vdots \\
0 & 0 & 0 & 0 & \cdots & 0 & 1 \\
-1 & -1 & -1 & -1 & \cdots & -1 & -1
\end{array}\right|
$$

and

$$
\pi_{2}=\left|\begin{array}{cccccc}
0 & 1 & 0 & 0 & \cdots & 0 \\
0 & 0 & 1 & 0 & \cdots & 0 \\
\vdots & \vdots & \vdots & \vdots & \cdots & \vdots \\
0 & 0 & 0 & 0 & \cdots & 1 \\
1 & 0 & 0 & 0 & \cdots & 0
\end{array}\right|
$$

are respectively of periods $n+2$ and $n+1$. Indeed their characteristic equations are respectively

for $\pi_{1}$ :

$$
\lambda^{n+1}+\lambda^{n}+\cdots+\lambda+1=0 ;
$$

for $\pi_{2}$ :

$$
\lambda^{n+1}-1=0 \text {. }
$$

The collineations $\pi_{1}, \pi_{2}$ are then readily seen to satisfy the conditions of Theorem 1. This completes the proof of

Theorem 2. Rational normal curves $C_{n}$ exist which define in the way indicated discontinuous $\zeta$-groups containing elliptic substitutions of period $n+1$ or period $n+2$.

This result is of considerable interest on account of the fact that it proves for the first time that the class of $\zeta$-groups $\Gamma_{n}$ $(n>2)$ is indeed more extensive than the class of groups $\Gamma_{2}$. In particular the classes of groups $\Gamma_{3}$ and $\Gamma_{4}$ contain groups with elliptic substitutions of period 5 ; no extensive class of such groups have as yet been defined arithmetically. The detailed discussion of the groups defined by normal curves $C_{3}$ and $C_{4}$ is, therefore, greatly to be desired. This discussion doubtless would lead to results of much interest in themselves, and might point the way for a more detailed treatment of the general case, which at present seems to be of too great complexity for immediate treatment. In this connection it may be noted that the curves $C_{n}$ defined by equations (4) will lead to groups in which the 
elliptic substitutions of periods $n+1$ or $n+2$ have double points at $\zeta=0$ and $\zeta=\infty$. Their coefficients will therefore be imaginary. If, however, the curve $C_{n}$ is written

$$
Z_{i}=c_{i}(\zeta-\eta)^{n-i}(\zeta-\bar{\eta})^{i} \quad(i=0,1, \ldots, n),
$$

these double points will be at $\zeta=\eta, \zeta=\bar{\eta}$ respectively. It is, therefore, possible to write down at once the equations of a $C_{n}$ for which the corresponding $\Gamma_{n}$ contains any given elliptic substitution of period $n+1$ or $n+2$ of form (1).

It may also be noted that the period $\omega=n$, which satisfies the necessary condition of the earlier paper, is not in general possible if $n>2$. For $n=3$, for example, there exist no collineations in $S_{3}$ of period 3 which satisfy the conditions of Theorem 1 .

Finally we enquire regarding the existence of parabolic substitutions in a $\Gamma_{n}$. Supposing the coordinate system so chosen as to render the equations of the $C_{n}$ of the form

$$
z_{i}=\zeta^{n-i} \quad(i=0,1, \ldots, n),
$$

any parabolic substitution may be taken to be

$$
\zeta^{\prime}=\zeta+1 .
$$

The corresponding collineation leaving $c_{n}$ invariant is then readily calculated from the equations

$$
z_{i}^{\prime}=(\zeta+1)^{n-i} \quad(i=0,1, \cdots, n),
$$

and substituting from (5). The characteristic equation of this collineation is found to be

$$
(\lambda-1)^{n+1}=0 .
$$

This shows first that a collineation in $S_{n}$ which produces on an invariant $C_{n}$ a parabolic projectivity has only a single double point.*

It shows, furthermore, that any $C_{n}$ defining a $\Gamma_{n}$ with parabolic substitutions must contain a point with integral coordinates. This is a generalization of the corresponding fact for the case $n=2$. Fricke $\dagger$ used this in the case $n=2$ to show that every $\Gamma_{2}$ with parabolic substitutions must be commensurable with the elliptic modular group. His argument, however, does

* Cf. in this connection the footnote on p. 365 .

$\dagger$ Fricke-Klein, Automorphe Functionen, vol. 1, p. 518. 
not seem to apply when $n>2$. This suggests the important question: Can any fundamental circle group (discontinuous $\zeta$-group that is representable with real coefficients) be defined arithmetically as a $\Gamma_{n}$ for a sufficiently high value of $n$ ?

UNIVERSITY OF ILLINOIS, December, 1909.

\section{A NEW ANALYTICAL EXPRESSION FOR THE NUMBER $\pi$, AND SOME HISTORICAL CONSIDERATIONS.}

BY DR. G. VACCA.

IN the common exposition of the history of mathematics, more attention is given to results than to methods, and it is only rarely that old theorems and demonstrations are translated into the modern and living mathematical language. The great mathematicians of the eighteenth century (Euler, Lagrange, and others) have made important contributions to this difficult work. It seems to me that even to-day something can be done in this direction, and many new results can be obtained by a careful reading of the ancient classics of mathematics. The following is an example:

Let $f(x)$ be a function defined by the relation

$$
f(x)=\frac{1}{2}(x+|x|)
$$

and suppose that $f^{n}(x)$ means the result of the operation $f$ applied $n$ times to the number $x$. Then we have, if $i=\sqrt{ }-1$,

$$
{ }_{\pi}^{2}=\lim _{n=\infty} f^{n}(i) .
$$

This elegant theorem can be easily proved. It gives a possible analytical definition of the number $\pi$.

It is now to be observed that this new formula is only the analytical expression (using the geometrical representation of complex quantities of Gauss) of a series of points (approaching the vertex) of the quadratrix ( $\tau \epsilon \tau \rho a \gamma \omega \nu i \zeta o v \sigma a)$ of Deinostratos. ${ }^{*}$

We may try to transform the second member of (1) into a real expression. This can be done by elementary methods. But the result is nothing else than the well-known infinite product of complicated quadratic radicals first given by Vieta. $\dagger$

* Pappi Coll. Math., lib. IV, prop. 25.

† Vieta, Opera, ed. Schooten, Lugd. Batav., 1646, p. 400. 